
\title{
3 Research Square \\ Factors Influencing Left Ventricular Ejection Fraction in patients with CMVD and OCAD
}

\section{Henry Anselmo Mayala ( $\nabla$ henrymayala@yahoo.co.uk)}

JAKAYA KIKWETE CARDIAC INSTITUTE (JKCI) https://orcid.org/0000-0001-9677-2520

\section{Mafuru Magesa}

Huazhong University of Science and Technology

\section{Abdalah Mkangala}

Jakaya Kikwete Cardiac Institute

\section{Mark Mayala}

Jakaya Kikwete Cardiac Institute

\section{Pedro Pallangyo}

Jakaya Kikwete Cardiac Institute

\section{Dickson Minja}

Jakaya Kikwete Cardiac Institute

Mohamed Janabi

Jakaya Kikwete Cardiac Institute

\section{Wang Zhao-hui}

Huazhong University of Science and Technology Tongji Medical College

\section{Research note}

Keywords: Coronary artery microvascular dysfunction (CMVD), Coronary flow reserve (CFR), Left ventricular ejection fraction (LVEF), LDL-c, BNP

Posted Date: February 14th, 2020

DOI: https://doi.org/10.21203/rs.2.23558/v1

License: (c) (i) This work is licensed under a Creative Commons Attribution 4.0 International License.

Read Full License

Version of Record: A version of this preprint was published at BMC Research Notes on March 16th, 2020. See the published version at https://doi.org/10.1186/s13104-020-05008-2. 


\section{Abstract}

Objective: The aim of our research was to evaluate the relationship involving LVEF, LDL, BNP, Troponin I and CFR, and to determine the predictors of LVEF in patients with CMVD and OCAD, and in patients with CMVD. Results: The mean age was $58.5 \pm 12.5$ years. Approximately $60 \%$ of the patients were women. Chest pain was the common symptom in both conditions around $45 \%$ followed by chest tightness which was $25 \%$. In patients OCAD and CMVD we found low density lipoprotein-c (LDL-C) had significant inverse relationship with LVEF ( $r=-0.323, P=0.042)$, LVEF also had significant negative relationship with $B N P$, and Troponin-I. While a significant direct relationship turned out to be observed linking LVEF with CFR ( $r=$ $0.422, P=0.007)$. Left ventricular ejection fraction had significant negative relationship with LDL-C $(r=$ $-0.489, P=0.029)$, and BNP ( $r=-0.472, P=0.035)$ in patients with OCAD only. Age, blood pressure, lipid levels, RDW, HbA1C, symptoms, NYHA classification, Alcohol drinking, hypertension, diabetes mellitus, troponin levels and BNP were the predictors for LVEF in CMVD patients. We depicted a strong negative relationship between LVEF and biomarkers (LDL-C, BNP, Troponin-I), with a significant positive association between LVEF and CFR.

\section{Introduction}

Coronary artery disease is a major non communicable disease problem around the world. The coronary vessels damage can be caused by an array of crucial risk factors such as hypertension, dyslipidemia, diabetes mellitus and smoking cigarettes [1]. Furthermore, obstructive coronary artery disease occurs less frequently in females, preceding studies revealed a poor prognosis in females in relation to male counterparts amid patients exhibiting with symptoms of stable angina [2]. Even though there is detailed documentation and guidelines emphasizing administration of secondary prevention medication, an under usage of these medication was observed, meaning that many patients with coronary artery disease did not attain the treatment objective for secondary prevention [3]. Epidemiology studies have revealed men to be more affected by OCAD compared to women. Moreover, female patients are believed to have more symptom burden and a higher rate of functional disability but a lower prevalence of OCAD, they usually present with NOCAD particularly CMVD. In patients with CAD, men tend to have a higher lipid core compared with women [4].

Previous studies were done to investigate serum total cholesterol in relation to LVEF and Coronary artery disease where they found out higher total cholesterol and HDL-C are associated with higher LVEF $[5,6]$. There were no studies done in evaluating the LDL-C, BNP, Troponin-I and CFR in association with LVEF in patients with OCAD and CMVD.

In our study, we used a prospective clinical observational design to investigate the association between LVEF and LDL-C, BNP, Troponin-I, and CFR and to determine the predictors of LVEF

\section{Methodology}




\section{Study population:}

We recruited 40 patients attending to our hospital for the first time divided into two sub-group where by half had coronary microvascular disease and the other half had obstructive coronary artery disease.

\section{Study Design:}

A prospective clinical observational study

\section{Inclusion Criteria:}

- ST-T dynamic variations on ECG (ST segment desolation, symmetrical T wave reversion, or dynamic change that appears at the time when the chest discomfort occurs)

- Coronary artery examination by coronary angiography was accomplished

\section{8-79 years of age}

We recruited patients who were showing up for the initial appointment to our medical institution and not in any treatment at all.

\section{Exclusion Criteria:}

Acute myocardial infarction

Patients who had percutaneous coronary intervention and those who had coronary artery bypass graft A further cardiac disorders affecting ventricular wall motion or cardiac ejection function, such as stress cardiomyopathy, hypertrophic cardiomyopathy, dilated cardiomyopathy, myocarditis, myocardial amyloidosis

Severe arrhythmias such as permanent atrial fibrillation, recurrent and poorly controlled ventricular arrhythmias

Severe valvular heart disease

Follow up patients on medications including statins

Allergic habitus

Patients or their family members refused to participate in the study.

\section{Definition Of Terms}

CMVD-ST- segment depression or T-wave inversion on ECG but had TIMI 3 flow on Coronary angiography OCAD-ST- segment depression or T-wave inversion on ECG and either TIMI I or II flow on CAG

Study Objective:

The intention of this research was evaluating the relationship between LVEF and LDL, BNP, Troponin I and CFR, and to determine the predictors of LVEF in patients with CMVD and OCAD, and in patients with 
CMVD.

Image Acquisition

PET-CT scan was used to measure coronary flow reserve and assess the microvascular coronary perfusion. The images were obtained using a dedicated PET/CT scanner (Discovery VCT ${ }^{\circledR}$, GE medical systems, Milwaukee WI, USA) immediately after intravenous injection of 3.75 to $5.55 \mathrm{MBq} / \mathrm{kg}$ of $13 \mathrm{~N}$ $\mathrm{NH} 3$, rest and ATP-stressed respectively.

A cutoff CFR value for our study was 2.6 [7].

Statistical Analysis Software:

The statistical analysis was done using IBM SPSS Statistics for Windows, Version 20.0. Armonk, NY: IBM Corp, U.S.A

Statistical analysis:

Baseline patient characteristics were summarized. All data are presented as mean \pm SD for continuous variables and $\mathrm{n}$ (\%) for categorical variables. Comparisons between groups were made using Pearson correlation or Spearman's rho for continuous variables and Fisher exact test for categorical variables. A Pvalue of $<0.05$ was considered statistically significant. A multivariate linear regression model was done to determine the predictors of LVEF.

\section{Results}

\section{Patients Demographic and clinical characteristics}

Forty patients participated in our study, whereby twenty patients had coronary microvascular dysfunction (CMVD) and twenty patients had obstructive coronary artery disease (OCAD). The mean age was $58.5 \pm$ 12.5 years. Approximately $60 \%$ of the patients were women. The mean left ventricular ejection fraction was $56.7 \pm 7.9$, and the mean coronary flow reserve was $2.04 \pm 0.56$ respectively. The patient's demographic and clinical characteristics are summarized in Table 1. 
Table 1

Demographics and Clinical Characteristics of Patients $(n=40)$

Variables

Age $($ mean $\pm S D)$

Gender n (\%)

- Male

- Female

Symptoms n (\%)

- Chest pain

- Chest tightness

- Difficulty in breathing

- mixed symptoms

NYHA classification $\mathrm{n}(\%)$

- Class I

- Class II

- Class III

- Class IV

Smoking, $\mathrm{n}(\%)$

Alcohol, n (\%)

Hypertension, $\mathrm{n}(\%)$

Diabetes mellitus, $\mathrm{n}(\%)$

Systolic BP in $\mathrm{mmHg}$, (mean $\pm \mathrm{SD}$ )

Diastolic BP in $\mathrm{mmHg}$, (mean $\pm \mathrm{SD})$

LDL-C in $\mu \mathrm{mol} / \mathrm{I}$, (mean \pm SD)

$\mathrm{HDL}-\mathrm{C}$ in $\mu \mathrm{mol} / \mathrm{l},($ mean $\pm \mathrm{SD})$

Troponin I in pg/ml, (mean \pm SD)

Red cell distribution width, $\mathrm{n}(\%)$
Value

$58.5 \pm 12.5$

$16(40)$

$24(60)$

$18(45)$

$10(25)$

8 (20)

$4(10)$

$20(50)$

12 (30)

$6(15)$

2 (5)

$20(50)$

$18(45)$

$26(65)$

$16(40)$

$136.6 \pm 20.9$

$82.6 \pm 16.6$

$3.5 \pm 1.6$

$1.16 \pm 0.4$

$18.9 \pm 16.5$

$15.5 \pm 3.2$

LDL-C, low density lipoprotein- cholesterol, HDL-C, high density lipoprotein-cholesterol, BNP, brain natriuretic peptide, SD, standard deviation 
Glycated Hemoglobin

$\mathrm{BNP}$ in $\mathrm{pg} / \mathrm{ml}$, (mean $\pm \mathrm{SD})$

Left ventricular ejection fraction

Coronary flow reserve
$6.3 \pm 1.8$

$113.3 \pm 167.8$

$56.7 \pm 7.9$

$2.04 \pm 0.56$

LDL-C, low density lipoprotein- cholesterol, HDL-C, high density lipoprotein-cholesterol, BNP, brain natriuretic peptide, SD, standard deviation

\section{Relationship between left ventricular ejection fraction (LVEF), LDL-C, BNP, troponin-I and CFR in patients with CMVD and OCAD}

We hypothesized that the factors influencing left ventricular ejection fraction for patients with CMVD and OCAD to be LDL-C, BNP, Troponin-I and CFR. We conducted correlation tests using Spearman's rho to assess the relationship between LVEF, LDL-C, BNP, Troponin-I and CFR in patients with CMVD and OCAD (Fig. 1A, B, C and D). Low density lipoprotein-C (LDL-C) had significant inverse relationship with LVEF ( $r=$ $-0.323, P=0.042)$, LVEF also had significant negative relationship with $B N P(r=-0.562, P<.0001)$, and troponin-I $(r=-0.311, P=0.04)$. While a significant positive relationship was observed between LVEF and CFR $(r=0.422, P=0.007)$.

\section{Relationship between left ventricular ejection fraction (LVEF), LDL-C and BNP in patients with OCAD}

Left ventricular ejection fraction had significant negative relationship with LDL-C, and BNP. We observed fewer factors influencing the left ventricular ejection fraction when we separated OCAD from CMVD patients, and there was no correlating factor in CMVD subgroup.

\section{Determination of predictors of LVEF}

i)

In patients with CMVD and OCAD

A backward multivariate linear regression model was done for determination of predictors of LVEF in patients with CMVD and OCAD. In this study, the variables Age, symptoms, NYHA classification and BNP qualified to enter the model. After adjusting for confounders, the patients age (coefficient $\beta=0.19,95 \% \mathrm{Cl}$, 39.5-58.6, $P=0.023)$, Difficulty in breathing (coefficient $\beta=-6.95,95 \% \mathrm{Cl},(-11.9)-(-2.0), P=0.007)$, NYHA 
Class III (coefficient $\beta=-7.14,95 \% \mathrm{Cl},(-12.55)-(-1.74), P=0.011)$, NYHA Class IV (coefficient $\beta=-17.25$, $95 \% \mathrm{Cl},(-26.18)-(-8.32), \mathrm{P}<.0001)$, and BNP (coefficient $\beta=-0.03,95 \% \mathrm{Cl},(-0.042)-(-0.019), \mathrm{P}<.0001)$ were determined as predictors of LVEF in patients with CMVD and OCAD.

ii)

In patients with CMVD

After adjusting for confounders, the patients age (coefficient $\beta=1.31,95 \% \mathrm{Cl}, 1.07-1.55, \mathrm{P}<.0001$ ), systolic blood pressure (coefficient $\beta=-0.58,95 \% \mathrm{Cl},(-0.76)-(-0.41), P<.0001)$, diastolic blood pressure (coefficient $\beta=-1.71,95 \% \mathrm{Cl},(-2.14)-(-1.28), P<.0001), \mathrm{HDL}$ (coefficient $\beta=-4.8,95 \% \mathrm{Cl},(-8.5)-(1.14), \mathrm{P}=$ 0.02 ), HbA1c (coefficient $\beta=2.69,95 \% \mathrm{Cl}, 1.66-3.74, P=0.001$ ), Chest tightness (coefficient $\beta=33.3,95 \%$ $\mathrm{Cl}, 26.4-40.2, \mathrm{P}<.0001$ ), difficulty in breathing (coefficient $\beta=12.3,95 \% \mathrm{Cl}, 6.93-17.7, \mathrm{P}=0.002$ ), NYHA class I (coefficient $\beta=20.4,95 \% \mathrm{Cl}, 13.3-27.5, P=0.001$ ), alcohol (coefficient $\beta=20,95 \% \mathrm{Cl}, 15.6-24.6, P$ $<.0001$ ), Hypertension (coefficient $\beta=57,95 \% \mathrm{Cl}, 47-67, \mathrm{P}<.0001$ ), Diabetes mellitus (coefficient $\beta=-64$, $95 \% \mathrm{Cl},(-77)-(-51), \mathrm{P}<.0001)$, Troponin I (coefficient $\beta=-1.65,95 \% \mathrm{Cl},(-1.9)-(-1.3), \mathrm{P}<.0001)$, and BNP (coefficient $\beta=0.35,95 \% \mathrm{Cl}, 0.24-0.46, P=0.001$ ) were determined as predictors of LVEF in patients with CMVD (Table 2). 
Table 2

Multivariate linear regression model investigating the predictors of LVEF in patients with CMVD $(n=20)$

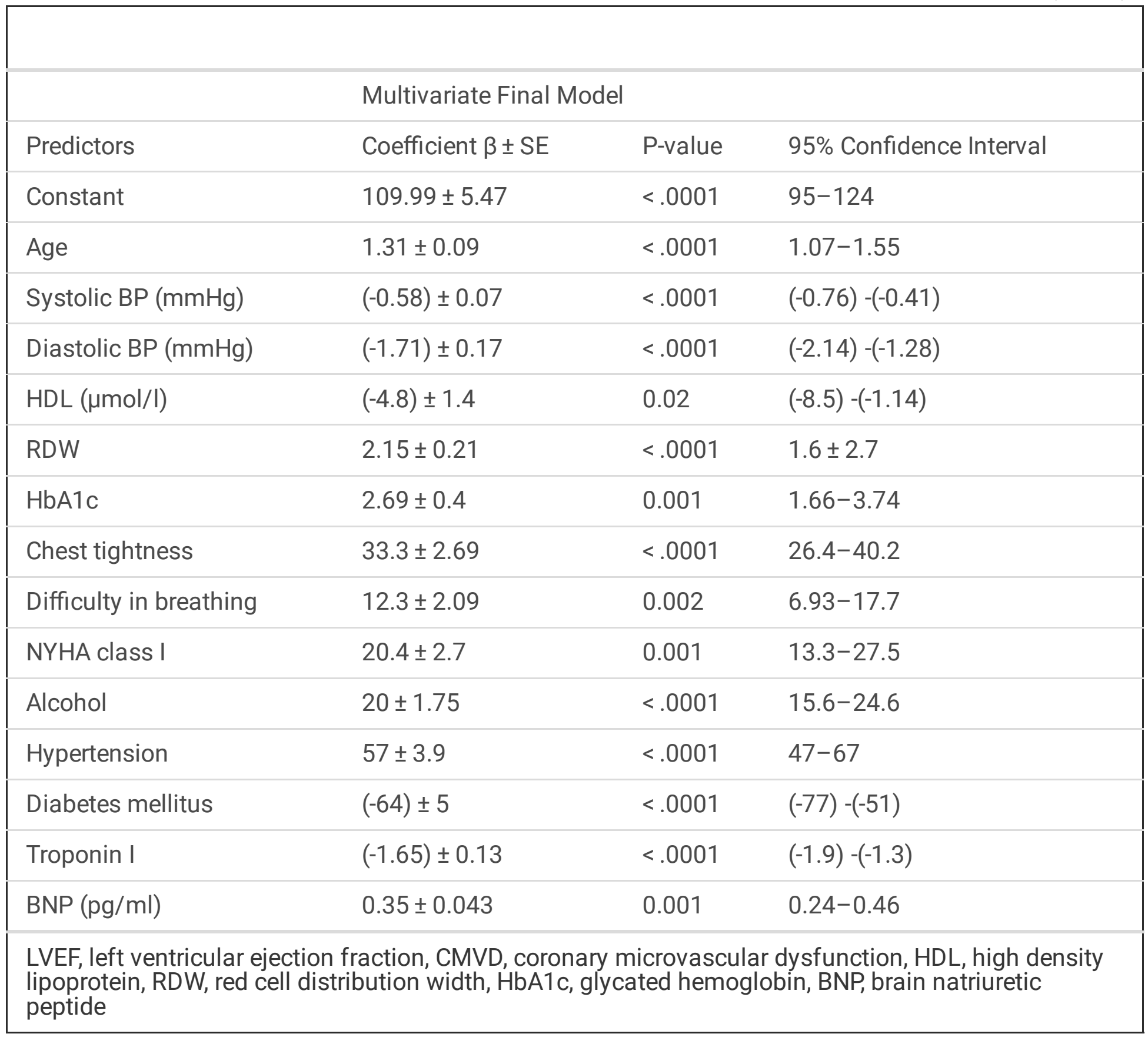

\section{Discussion}

The current research indicated a negative correlation between LVEF and LDL-C, BNP and Troponin-I in patients with OCAD and CMVD. To the best our knowledge, this is the first research to reveal the relationship between LVEF and biomarkers (LDL-C, BNP and Troponin-I). We also found out a positive association between LVEF and CFR in patients with OCAD AND CMVD. This finding was congruent with our previous study, whereby there was a positive correlation between LVEF and CFR [8].

Previous studies have shown that hyperlipidemia adversely influenced the left ventricular ejection fraction, particularly in patients with myocardial infarction. They went further and detailed an important 
positive correlation between left ventricular ejection fraction and high density lipoprotein-cholesterol, suggesting that HDL-cholesterol might influence left ventricular systolic function through extraatherosclerotic mechanisms because they observed left ventricular ejection fraction was adversely influenced by dyslipidemia irrespective of the severity of coronary atherosclerosis $[9,10,11]$. There was another study in which, they investigated the association between lipid profile levels and right ventricular volume overload in congestive heart failure, where they revealed lipid levels (total cholesterol, triglycerides, low density lipoprotein-c, and high density lipoprotein-c) were inversely correlated to right ventricular end diastolic diameter and right atrium [12]. In our study, which involved patients with obstructive coronary artery disease and coronary microvascular dysfunction, we found out LDL-c to be inversely correlated with left ventricular ejection fraction and it was statistically significant. Meaning oneunit change increase in LDL-c is associated with a unit decrease in LVEF percentage. This show our study concur with previous study findings.

Several studies have revealed a negative correlation between BNP and left ventricular ejection fraction. They revealed that BNP levels were low in patients with heart failure with preserved ejection fraction compared to patients with heart failure with reduced ejection fraction. In another study, they also discovered an important correlation between NT-proBNP and LVEF in elderly patients, whereby worsening LVEF had a significant correlation with NT-proBNP levels $[13,14]$. In our study, we also found a negative correlation between left ventricular ejection fraction and B-type natriuretic peptide in patients with obstructive coronary artery disease and coronary microvascular dysfunction $(r=-0.562, P<.0001)$ and in patients with OCAD alone $(r=-0.472, P=0.035)$, meaning that one unit change increase in BNP was related with a unit decrease in LVEF percentage.

Furthermore, another biomarker troponin-l is a power indicator of myocardial necrosis, it has been studied before and was found to be inversely correlated with left ventricular ejection fraction especially in patients after first myocardial infarction. They found out that the left ventricular ejection fraction of $<50 \%$ was predicted by troponin I concentration of $>6.6 \mathrm{ng} / \mathrm{ml}$. In another research analysis, it showed that patients with severe left ventricular systolic dysfunction (LVEF $<35 \%)$ had the highest level of troponin I and vice versa. They also showed that the LVEF had a negative correlation with troponin I levels $(r=-0.54$, $P=0.001)$. Despite the above fact, there was an exploration of troponin $T$, whereby it was also revealed that there was a negative correlation between troponin T levels and LVEF $(r=-0.72, P=<.0001)[15,16$, 17]. In our findings, we showed that there was a significant negative relationship between troponin I and left ventricular ejection fraction in patients with OCAD and CMVD $(r=-0.311, P=0.04)$. Meaning one-unit change increase in troponin I levels was associated with a unit decrease in LVEF percentage.

Form our study, we discovered the importance of these biomarkers (LDL-C, BNP, and Troponin-I) as prognostic indicators for patients with OCAD and CMVD.

Moreover, there were studies done in the area of determination of predictors of LVEF. Whereby, their analysis demonstrated that several traditional and easily available factors were associated with a greater risk of heart failure development, even among low-risk CAD population. Some of the studies showed 
lipids predicted the severity of new onset CAD in type-2 DM patients and not in relation to LVEF, differentiating from our study. In another research, low HDL-c was strongly predictive of cardiovascular events in patients with coronary artery disease $[18,19$, and 20]. In our research we also found out that traditional risk factors influenced the LVEF concurring the previous studies even though we were the only one, who further evaluated patients with OCAD and CMVD. In the current published articles indicated the relevance of inflammatory biomarkers particularly CRP and Pentraxin 3 as the prognostic indicators of coronary artery disease congruent with our findings even though we assessed different biomarkers (LDLc, BNP, troponin-I) [21,22].

We believe by sharing these findings of our study, will empower the clinicians with knowledge on coronary microvascular dysfunction in relation to OCAD, by exploring the relationship between LVEF and biomarkers, and predictors of LVEF in these patients.

\section{Conclusion}

There was a strong negative relationship between LVEF and biomarkers (LDL-C, BNP, Troponin-I), with a significant positive association between LVEF and CFR. Traditional risk factors were the predictors of LVEF in OCAD and CMVD patients.

Limitation

a small sample size was our study limitation

\section{Declarations}

\section{Ethical approval and consent to participate:}

The clinical protocol and the informed consent forms were approved by the ethics committee of Tongji medical college of Huazhong University of science and technology. All patients read and signed the published. informed consent. This clinical study was conducted according to the revised declaration of Helsinki concerning biomedical research in using patient information. All authors agreed for this manuscript to be published.

\section{Consent for publication}

Not applicable

\section{Availability of Data and materials}

Data and materials are available upon request to the authors

\section{Competing Interests}

The authors declare that they have no competing interests 


\section{Funding:}

This study did not receive any funding

\section{Authors Contribution:}

Drafting of manuscript was done by: $H A M, M M, A M, M M, P G, D M, M J$, WZ. Critical revision and correction were done by: WZ. All authors read and approved the final manuscript.

\section{Acknowledgements}

We thank the Staffs and management of Union Hospital of Huazhong University of Science and Technology for granting permission to conduct this study. And we thank all patients who participated in the study

\section{Abbreviations:}

LVEF- left ventricular ejection fraction, LDL-C- low density lipoprotein-cholesterol, BNP-B-type natriuretic peptide, CFR-coronary flow reserve, CMVD-coronary microvascular disease, and OCAD- obstructive coronary artery disease.

\section{Author Details:}

1, 3,4, 5, 6, 7 Jakaya Kikwete Cardiac Institute, P. O. Box 65141 Dar es Salaam, West Upanga, Kalenga Street, Ilala District, Dar es Salaam Region. Tel: +255-22-2152392 / +255 755144363 Mob : +255-782042019. Email: info@jkci.or.tz

2, 8 Huazhong university of science and technology, 1037 Luoyu Road, Wuhan, Hubei, China, Post Code: 430074, Tel: +862787542457 Email: admission@hust.edu.cn

\section{Data Sharing}

For data sharing for this research paper, please contact the author by email: mayalahenry29@gmail.com

\section{References}

1. Gander J, Sui X, Hazlett LJ, et al. Factors related to coronary heart disease risk among men: validation of the Framingham risk score. Prev Chronic Dis. 2014; 11: E 140

2. Zhang $Y$, Liu Y, Zhang $\mathrm{H}$, et al. Impact of sex specific differences in calculating the pretest probability of obstructive coronary artery disease in symptomatic patients. Coronary artery disease, 30(2): 124130

3. Jotveit J, Halvorsen S, Kaldal A, et al. Unsatisfactory risk factor control and high rate of new cardiovascular events in patients with myocardial infarction and poor coronary artery disease. BMC Cardiovasc Dis 2019; 19: 71 
4. Tian J, Wang X, Tian J, and Yu B. Gender difference in plaque characteristics of non-culprit lesions in patients with coronary artery disease. BMC Cardiovasc Dis 2019; 19:45

5. Liu Y, Hao Z, Xiao C, et al. Association of serum total cholesterol and left ventricular ejection fraction in patients with heart failure caused by coronary heart disease. Arch Med Sci. 2018; 14(5): 988-994

6. Zhao Q, Li J, Yang J, et al. Association of total cholesterol and HDL-c levels and outcome in coronary heart disease patients with heart failure. Medicine 2017; 96(6): e6094

7. Loffler and Bourque. Coronary microvascular dysfunction, microvascular angina, and management. Curr Cardiol Rep. 2016; 18(1): 1

8. Mayala HA, Bakari KH, Mghanga FP, and ZhaoHui W. Clinical significance of PET-CT Coronary flow reserve in diagnosis of non-obstructive coronary artery disease. BMC Res notes 2018; 11:566

9. Wang TD, Wu CC, Chen WJ, et al. Dyslipidemia have a detrimental effect on left ventricular systolic function in patients with a first myocardial infarction. Am J Cardiol. 1998; 81:531-537

10. Kempen HJ, Van Gent CM, Buytenhek R, et al. Association of cholesterol concentrations in lowdensity lipoprotein, high density lipoprotein, and high-density lipoprotein subfractions, and of apolipoproteins Al and All, with coronary stenosis and left ventricular function. J Lab Clin Med. 1987; 109: $19-26$

11. Wang TD, Lee CM, Wu CC, et al. The effects of dyslipidemia on left ventricular systolic function in patients with stable angina pectoris. Atherosclerosis 1999; 146:117-124

12. Chen $\mathrm{Y}, \mathrm{He} X \mathrm{X}, \mathrm{Meng} \mathrm{H}$, et al. Relationship between lipid levels and right ventricular volume overload in congestive heart failure. J Geriatr Cardiol 2014; 11: 192-199

13. Leto L, Testa M, and Feola M. Correlation between B-type natriuretic peptide and functional/cognitive parameters in discharged congestive heart failure patients. International journal of endocrinology, volume 2015, article ID 239136

14. Belagavi AC, Rao M, Pillai A, and Srihari US. Correlation between NT-proBNP and left ventricular ejection fraction in elderly patients presenting to emergency department with dyspnea. Indian Heart journal 2012; 6403:302-304

15. Somani D, Gahlot RS, Lakhotia M, et al. Troponin I measurement after myocardial infarction and its correlation with left ventricular ejection fraction: A prospective study. JIACM 2005; 6(1): 38-41

16. Khan MH, Islam MN, Aditya GP, et al. Correlation of troponin-I level with left ventricular ejection fraction and in hospital outcomes after first attack of non-ST segment elevation myocardial infarction. Mymensingh Med J 2017; 26(4):721-731

17. Rao ACR, Collinson PO, Canepa-Anson R, and Joseph SP. Troponin T measurement after myocardial infarction can identify left ventricular ejection fraction of less than $40 \%$. Heart $1998 ; 80: 223-225$

18. Lewis EF, Solomon SD, Jablonski KA, et al. Predictors of heart failure in patients with stable coronary artery disease: A PEACE study. Circ Heart Fail. 2009; 2(3): 209-216

19. Du Y, Chen J, Chen MH, et al. Relationship of lipid and lipoprotein ratios with coronary severity in patients with new on-set coronary artery disease complicated with type 2 diabetics. J Geriatr Cardiol 
2016; 13: 685-692

20. Miller M, Saidler A, Kwiterovich PO, and Pearson TA. Long term predictors of subsequent cardiovascular events with coronary artery disease and desirable levels of plasma total cholesterol. Circ. 1992; 86:1165-1170

21. Altay S, Gurdogan M, Keskin M, et al. The Inflammation-Based Glasgow Prognostic Score as a prognostic factor in patients with intensive cardiovascular care unit. Medicina (Kaunas). 2019; 55(5): 139

22. Altay $\mathrm{S}$, Cakmak H, Oz T, et al. Long term prognostic significance of pentraxin-3 in patients with acute myocardial infarction: 5- year prospective cohort study. Anatol J Cardiol. 2017; 17(3): 202-209.

\section{Figures}
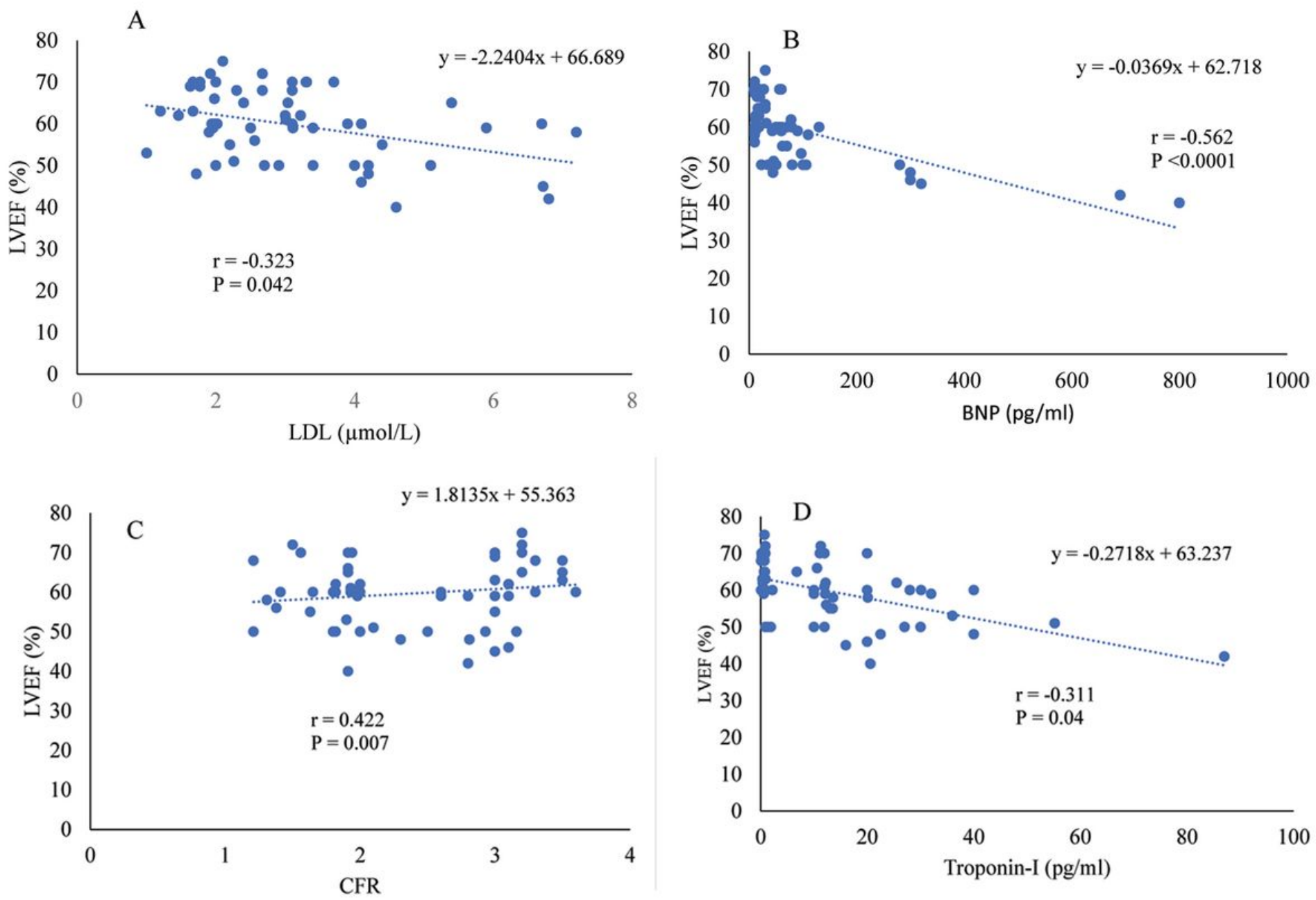

\section{Figure 1}

A: showing a negative correlation between LVEF (\%) and LDL-C $(\mu \mathrm{mol})$, B: showing a negative relationship between LVEF (\%) and BNP (pg/ml), C: showing a positive correlation between LVEF (\%) and CFR, and lastly D: showing a negative relationship between LVEF (\%) and Troponin I (pg/ml) 
Page 14/14 